\title{
Silencing of IncRNA SNHG12 inhibits proliferation and migration of vascular smooth muscle cells via targeting miR-766-5p/EIF5A axis
}

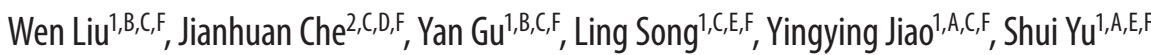 \\ ${ }^{1}$ Department of Cardiovascular Medicine, The First Hospital of Jilin University, China \\ ${ }^{2}$ Department of Oral and Maxillofacial Surgery, Hospital of Stomatology, Jilin University, China \\ A - research concept and design; $\mathrm{B}$ - collection and/or assembly of data; $\mathrm{C}$ - data analysis and interpretation; \\ $D$ - writing the article; $E$ - critical revision of the article; $F$ - final approval of the article
}

\section{Address for correspondence}

Shui Yu

E-mail: qinlei256@163.com

Funding sources

None declared

Conflict of interest

None declared

Received on September 8, 2020

Reviewed on September 26, 2020

Accepted on February 19, 2021

Published online on May 20, 2021

Cite as

Liu W, Che J, Gu Y, Song L, Jia Y, Yu S. Silencing of IncRNA SNHG12 inhibits proliferation and migration of vascular smooth muscle cells via targeting miR-766-5p/ElF5A axis. Adv Clin Exp Med. 2021;30(6):591-598.

doi:10.17219/acem/133496

DOI

10.17219/acem/133496

Copyright

Copyright by Author(s)

This is an article distributed under the terms of the

Creative Commons Attribution 3.0 Unported (CC BY 3.0)

(https://creativecommons.org/licenses/by/3.0/)

\begin{abstract}
Background. Although long non-coding RNAs (IncRNAs) have been reported to serve as potential biomarkers of atherosclerosis (AS), the role of IncRNA small nucleolar RNA host gene 12 (SNHG12) in AS still remains to be elucidated.
\end{abstract}

Objectives. The present study aimed to investigate the regulatory effects and potential mechanisms of SNHG12 in human vascular smooth muscle cells (hVSMCs).

Materials and methods. Reverse-transcription quantitative polymerase chain reaction (RT-qPCR) was employed to determine the expression of SNHG12, miR-766-5p and eukaryotic translation initiation factor $5 \mathrm{~A}$ (EFFA) in oxidized low-density lipoprotein (ox-LDL)-induced hVSMCS. After transfection with short hairpin RNA (shRNA)-SNHG12, cell viability was estimated using the Cell Counting Kit-8 (CCK-8) assay. Wound healing and transwell assays were used for evaluating migratory capacities of hVSMCs. To further investigate the regulatory mechanisms, binding sites between SNHG12 and miR-766-5p, and EIF5A and miR-766-5p were predicted using starBase database and validated using luciferase reporter gene assays. Moreover, cell viability and migration were detected following EIF5A overexpression and SNHG12-knockdown.

Results. SNHG12 was significantly upregulated in ox-LDL-induced hVSMCs. SNHG12 silencing inhibited ox-LDL-induced proliferation and migration of hVSMCS. Moreover, SNHG12 acted as a sponge of miR-7665p, and miR-766-5p also interacted with EIF5A. EIF5A plasmids promoted the capacities of proliferation and migration in ox-LDL-induced hVSMCS. However, shRNA-SNHG12 counteracted the facilitation of EIF5A plasmids on hVSMCs biological behaviors.

Conclusions. Taken together, these findings demonstrated that silencing of SNHG12 blocks the proliferation and migration of hVSMCs via targeting the miR-766-5p/EIF5A axis.

Key words: migration, SNHG12, human vascular smooth muscle cells, miR-766-5p, eukaryotic translation initiation factor $5 \mathrm{~A}$ 


\section{Background}

Long non-coding RNAs (lncRNAs) are a heterogeneous class of non-coding RNAs greater than 200 nucleotides in length without protein-coding capacity. ${ }^{1}$ Recently, studies have found that lncRNAs emerge as crucial regulators of atherosclerosis (AS). ${ }^{2,3}$ Atherosclerosis is commonly recognized as a lipid-induced chronic inflammation of the vascular wall associated with activation and dysfunction of resident vascular cells ${ }^{4}$ and contributes to stenosis of internal arteries due to plaque accumulation. ${ }^{5}$ The number of lncRNAs was reported to be implicated in regulating cholesterol and lipid metabolism, and they also play diverse roles in a variety of atherosclerotic processes including cell proliferation, migration, inflammation, differentiation, and apoptosis. ${ }^{6}$

Small nucleolar RNA host gene 12 (SNHG12) is one of the classes of SNHGs. ${ }^{7}$ Studies revealed that SNHG12 regulates cell proliferation, migration, invasion, and metastasis in several cancers, ${ }^{8-12}$ indicating a potential target for cancer-directed interventions. ${ }^{13}$ Except for its role in cancers, SNHG12 could also ameliorate brain microvascular endothelial cell injury. ${ }^{14}$ To date, a number of well-studied lncRNAs gave us important clues about their potential for AS treatment. ${ }^{15}$ For instance, lincRNA-p21 is downregulated in atherosclerotic plaques of ApoE(-/-) mice, and it can suppress vascular smooth muscle cell (VSMC) proliferation and induce apoptosis. ${ }^{16} \mathrm{HIF} 1 \alpha$-AS1 regulates the proliferation and apoptosis of VSMCs. ${ }^{17}$ The expression of H19 is higher in serum of AS patients, ${ }^{18}$ serving as a potential biomarker for diagnosing AS. However, the status, biological function and regulatory mechanisms of SNHG12 in AS are still unknown.

\section{Objectives}

We examined the expression of SNHG12 in human VSMCs (hVSMCs) exposed to oxidized low density lipoprotein (ox-LDL) and evaluated the influence of SNHG12 on cell migration. Furthermore, the regulatory mechanisms of SNHG12 on hVSMCs were explored.

\section{Materials and methods}

\section{Cell lines and transfection}

The hVSMCs (Cell Bank of the Shanghai Institute of Cell Biology, Shanghai, China) were cultured in Dulbecco's modified Eagle's medium (DMEM; ProCell, Wuhan, China) containing 10\% fetal bovine serum (FBS; Gibco, Waltham, USA) under an atmosphere of $95 \%$ air and $5 \% \mathrm{CO}_{2}$ at $37^{\circ} \mathrm{C}$. The ox-LDL (Solarbio, Beijing, China) was used to stimulate hVSMCs for 48 h. miR-766-5p mimic, miR-NC (negative control), and 2 pairs of short hairpin RNA (shRNA)-SNHG12
(sh-SNHG12-1, sh-SNHG12-2) were obtained from GenePharma Co., Ltd. (Shanghai, China). Overexpression plasmids of EIF5A and the negative control were generated with the help of Sangon Biotech (Shanghai, China). Cells were collected $24 \mathrm{~h}$ following transfection, and transfection efficiency was evaluated using reverse-transcription quantitative polymerase chain reaction (RT-qPCR).

\section{RT-qPCR}

Total RNA was harvested (TRIzol ${ }^{\mathrm{TM}}$ Plus RNA Purification Kit; Invitrogen, Carlsbad, USA) and reverse transcribed into cDNA (M-MLV Reverse Transcriptase; Promega, Madison, USA). TaqMan MicroRNA Assay kit (Applied Biosystems; Thermo Fisher Scientific, Waltham, USA) was employed to quantify $m i R-766-5 p$, the relative expression of $m i R-766-5 p$ was normalized to U6, and others were normalized to GAPDH based on the $2^{-\Delta \Delta C t}$ method. ${ }^{19}$ The primers used in this study were as follows: SNHG12, forward: 5'-GTGATACTGAGGAGGTGAG-3' and reverse: 5'-CCTTCTGCTTCCCATAGAG-3'; EIF5A, forward: 5'-AGGCCATGGCAAAATAACTG-3' and reverse: 5'-GGGTGGGGAAAACCAAAATA-3'; GAPDH, forward: 5'-AGCCTCCCGCTTCGCTCTCTGC-3' and reverse: 5'-ACCAGGCGCCCAATACGACCAAA-3'; miR-766-5p, forward: 5'-TCGAGTACTTGAGATGGAGTTTT-3' and reverse: 5'-GGCCGCGTTGCAGTGAGCCGAG-3'; U6, forward: 5'-CTCGCTTCGGCAGCACA-3' and reverse: 5'-AACGCTTCACGAATTTGCGT-3'.

\section{Cell viability assay}

The hVSMCs were seeded into a 96-well plate, then cells were incubated with $10 \mu \mathrm{L}$ Cell Counting Kit-8 (CCK-8) solution (Beyotime, Jiangsu, China) at $24 \mathrm{~h}, 48 \mathrm{~h}$ and $72 \mathrm{~h}$. Absorbance values were recorded on a BioTek microplate reader (BioTek, Winooski, USA) at $450 \mathrm{~nm}$.

\section{Wound healing assay}

An amount of $1 \times 10^{5}$ of hVSMCs were plated into each well of a 12 -well plate. When $100 \%$ confluence was achieved, the culture medium was removed and drew straight from the plate using a $200 \mu \mathrm{L}$ plastic pipette. The sample was washed gently to remove the floating cells, then serumfree medium was added and maintained in the incubator for $24 \mathrm{~h}$. Samples were photographed at $0 \mathrm{~h}$ and $24 \mathrm{~h}$ under a microscope (Axioscope 5; Carl Zeiss, Oberkochen, Germany).

\section{Transwell migration assay}

For the transwell migration assay, serum-free media containing $5 \times 10^{4}$ of hVSMCs were seeded into the upper chamber of a 24 -well transwell filter with $8-\mu$ m pore size. The lower chamber was filled with media supplemented 
with $10 \%$ FBS. Cells were allowed to transgress through the porous filters for $24 \mathrm{~h}$ at $37^{\circ} \mathrm{C}$. Then, VSMCs were fixed with $4 \%$ paraformaldehyde for $20 \mathrm{~min}$. Cells that migrated through the pores of the filter were stained with $1 \%$ crystal violet for $30 \mathrm{~min}$. The images were photographed under a fluorescence microscope (BX51; Olympus Corp., Tokyo, Japan), and the number of migrated cells was calculated using Image software (National Institutes of Health, Bethesda, USA).

\section{Luciferase reporter gene assay}

SNHG12 or EIF5A sequences containing the wild-type (WT) binding site or mutated-type (Mut) binding site for miR-766-5p were synthesized by Vigorous Biotechnology Beijing Co. Ltd. (Beijing, China) and cloned into the pmiRGLO vector (Promega). Prior to transfection, cells were seeded into 24 -well plates $\left(5 \times 10^{3}\right.$ cells/well $)$ and cultured for $24 \mathrm{~h}$. Afterward, the WT or Mut of SNHG12 was transiently co-transfected with $m i R-766-5 p$ mimics or miR-NC using Lipofectamine 3000 reagent for another $48 \mathrm{~h}$. The firefly luciferase activity normalized to Renilla represented the value of relative luciferase activity. Likewise, EIF5A WT or Mut co-transfected with miR-766-5p mimic or miR-NC was similar to the above method.

\section{Western blotting}

Total protein from treated cells was extracted using a radio immunoprecipitation assay lysis buffer containing proteinase inhibitors (Beyotime). After the determination of protein concentrations, equal protein samples $(40 \mu \mathrm{g})$ were loaded on SDS-PAGE gels and transferred onto polyvinylidene difluoride (PVDF) membranes (Merck Millipore, Madison, USA). Then, the membranes were blocked with $5 \%$ non-fat milk for $2 \mathrm{~h}$ and incubated with primary antibodies against EIF5A and GAPDH (both obtained from Cell Signaling Technology, Inc., Danvers, USA) at $4^{\circ} \mathrm{C}$ overnight. Horseradish peroxidase (HRP)-conjugated antibody (Santa Cruz Biotechnology, Santa Cruz, USA) was used to incubate membranes for $2 \mathrm{~h}$ at room temperature. The blots were visualized using the Odyssey Infrared Imaging System (LI-COR Biosciences, Lincoln, USA) and subsequently quantified using ImageJ software v. 1.52r (National Institutes of Health).

\section{Statistical analyses}

All data were presented as the mean \pm standard deviation (SD). The results were analyzed using GraphPad Prism v. 6.0 (GraphPad Software, Inc., San Diego, USA). An unpaired student's t-test was employed to evaluate differences between 2 groups, and one-way analysis of variance (ANOVA) followed by Tukey's post hoc test was used for comparison of differences between 3 or more groups. A value of $\mathrm{p}<0.05$ was considered statistically significant.

\section{Results}

\section{Interference with SNHG12 inhibits proliferation and migration of ox-LDL-induced hVSMCs}

We first investigated the expression of SNHG12. The hVSMCs were stimulated with different concentrations of ox-LDL, and, as shown in Fig. 1A, ox-LDL could promote the expression of SNHG12 in a dose-dependent manner. A volume of $100 \mathrm{mg} / \mathrm{L}$ ox-LDL was considered an optimal concentration to induce the transcription of SNHG12. To elucidate the function of SNHG12 in hVSMCs, a loss-of-function study was performed via transfecting sh-SNHG12 into cells. It was identified that sh-SNHG12-1 presented a better outcome for silencing SNHG12 (Fig. 1B). Afterward, we estimated the cell viability of hVSMCs in the absence of SNHG12, and results showed that sh-SNHG12-1 transfection significantly inhibited the increased cell proliferation caused by ox-LDL stimulation (Fig. 1C). Moreover, the wound healing assay and transwell migration assay indicated that ox-LDL-triggered cell migration was overturned by silencing of SNHG12 (Fig. 1D-G). These results suggest that disturbing the expression of SNHG12 could inhibit the viability and migration of hVSMCs induced by ox-LDL.

\section{SNHG12 functions as a sponge of miR-766-5p}

The LncRNAs are considered competing endogenous RNAs (ceRNAs) to bind with miRNAs and modulate gene expression. ${ }^{20}$ Jia et al. demonstrated that $m i R$ 766-5p participated in cell proliferation, migration and invasion in colorectal cancer. ${ }^{21}$ Of note, binding sites between SNHG12 and miR-766-5p were predicted using starBase v. 2.0 (http://starbase.sysu.edu.cn) (Fig. 2A), and $m i R-766-5 p$ mimic was validated to be effective to elevate the expression of $m i R-766-5 p$ (Fig. 2B). The luciferase reporter gene assay demonstrated that miR-766-5p mimic inhibited luciferase activity in hVSMCs transfected with SNHG12-WT (Fig. 2C). Additionally, it was found that shSNHG12-1 elevated the expression of miR-766-5p (Fig. 2D). Moreover, the level of $m i R-766-5 p$ in hVSMCs treated with ox-LDL was notably decreased (Fig. 2E). Collectively, this data reveal that $m i R-766-5 p$ is remarkably downregulated in ox-LDL-treated hVSMCs, and SNHG12 directly targeted miR-766-5p.

\section{EIF5A is a direct target gene of miR-766-5p}

As mentioned above, SNHG12 directly targeted $m i R$ 766-5p and served as a ceRNA to bind with $m i R-766-5 p$. The ceRNA activity forms a large-scale cross-talk network among the transcriptome. The miRNAs are generally regarded as active regulatory elements which reduce 


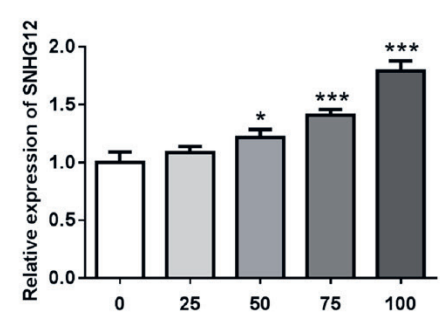

B

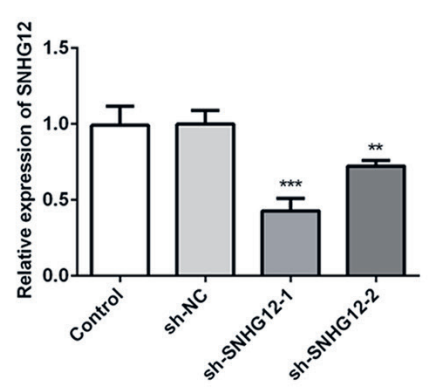

C

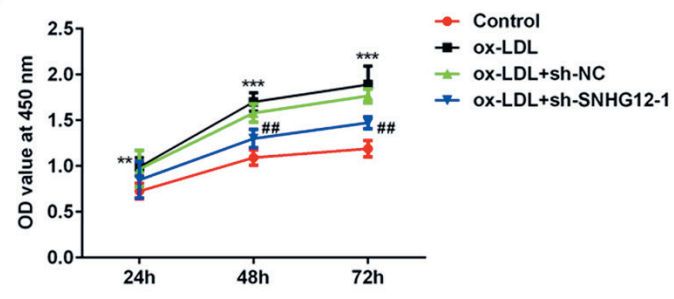

D
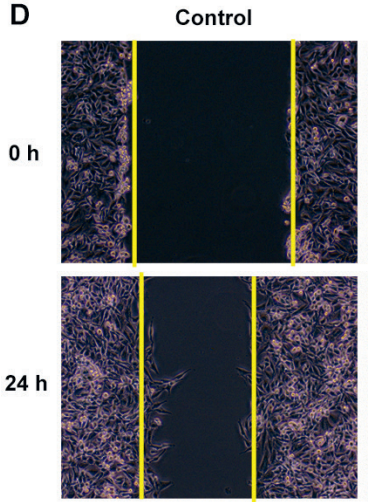

$\mathbf{F}$

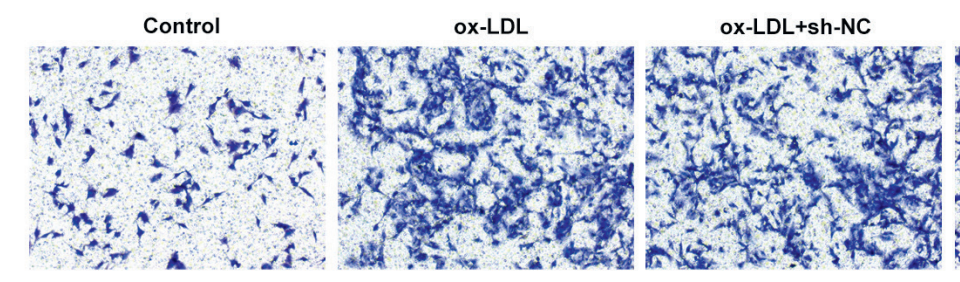

ox-LDL+sh-NC
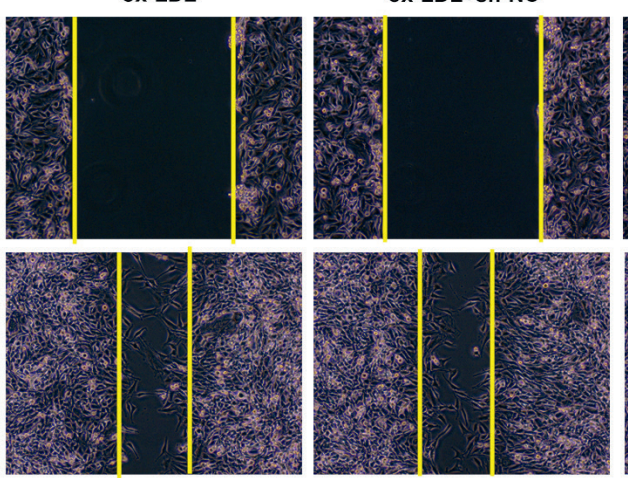

ox-LDL+sh-SNHG12-1

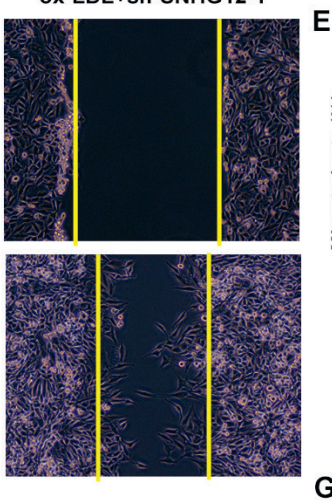

ox-LDL+sh-SNHG12-1
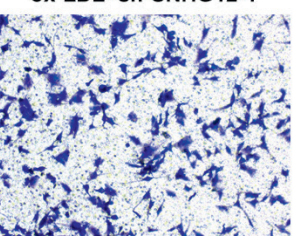
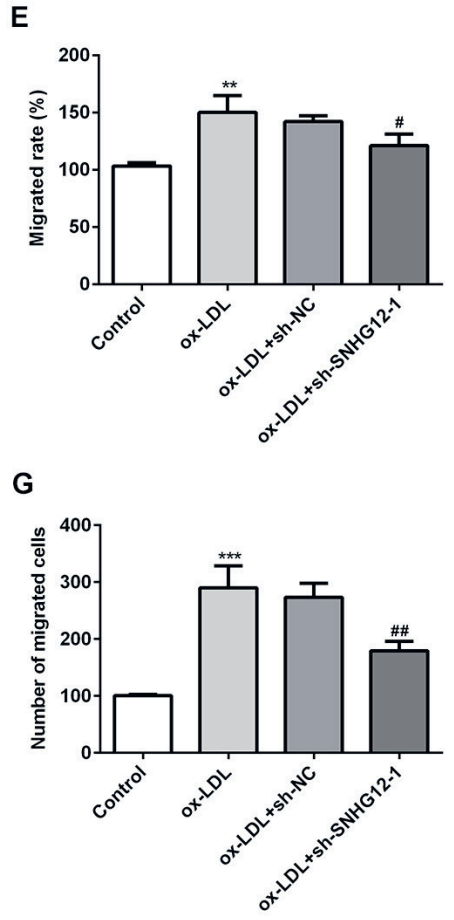

Fig. 1. Interference with SNHG12 inhibits proliferation and migration of hVSMCs

A. hVSMCs were stimulated with different concentrations of ox-LDL (0 mg/L, 25 mg/L, 50 mg/L, 75 mg/L, 100 mg/L), and the expression of SNHG12 was explored using RT-qPCR; ${ }^{*} p<0.05$, *** $<0.001$ compared to 0 mg/L ox-LDL; B. The expression of SNHG12 in hVSMCs transfected with sh-SNHG12-1 or sh-SNHG12-2 was estimated using RT-qPCR; ${ }^{* *} \mathrm{p}<0.01,{ }^{* * *} \mathrm{p}<0.001$ compared to the sh-NC group; C. hVSMCs were stimulated with 100 mg/L ox-LDL for $24 \mathrm{~h}, 48 \mathrm{~h}$ and $72 \mathrm{~h}$, and the cell viability was explored using CCK-8 assay; ${ }^{* *} \mathrm{p}<0.01,{ }^{* * *} \mathrm{p}<0.001$ compared to the control group; ${ }^{* \#} \mathrm{p}<0.01 \mathrm{compared}$ to the ox-LDL+sh-NC group. The capability of cell migration was assessed using wound healing assay (D and E) and transwell migration assay (F and G); ${ }^{* *} \mathrm{p}<0.01,{ }^{* * *} \mathrm{p}<0.001$ compared to the control group; $\mathrm{A}<0.05,{ }^{\# \#} \mathrm{p}<0.01$ compared to the ox-LDL+sh-NC group; $\times 100$ magnification.

the stability of target RNAs or inhibit their translation. ${ }^{22}$ Therefore, target mRNAs are considered as silencing objects of miRNAs. EIF5A is a small molecule protein in eukaryotic cells, which plays an important role in cell growth, survival and senescence. It is especially essential for cell proliferation. ${ }^{23}$ Of note, EIF5A was predicted as a potential target of $m i R-766-5 p$ (Fig. 3A). Luciferase reporter gene analysis was employed to test the potential interaction between them. It was observed that $m i R-766-5 p$ mimic apparently decreased the luciferase activity of EIF5A-WT in hVSMCs, and mutation of EIF5A abrogated the function of $m i R-766-5 p$ mimic (Fig. 3B). Subsequently, overexpression of $m i R-766-5 p$ reduced the transcription and translation of EIF5A (Fig. 3C,D). All of these data indicate that EIF5A may be a target mRNA of $m i R-766-5 p$.

\section{SNHG12 regulates the proliferation and migration of ox-LDL-induced hVSMCs via regulating EIF5A}

In order to further explore the interaction between EIF5A and SNHG12, gain-of-function and loss-of-function studies were applied in subsequent experiments. Overexpression plasmids were constructed and transfected into hVSMCs with or without sh-SNHG12-1, and high-expression of EIF5A validated the plasmids could overexpress EIF5A successfully. However, sh-SNHG12-1 drastically impeded the mRNA and protein levels of EIF5A (Fig. 4A,B). Cell viability was elevated in EIF5A overexpression group, while the effect was abolished by knockdown of SNHG12 (Fig. 4C). Migratory capacity represented by wound width illustrated that EIF5A promoted hVSMCs migration, while 
A

\begin{tabular}{|c|c|c|c|c|c|c|c|}
\hline hsa-miR-766-5p & ENSG00000197989 & SNHG12 & antisense & $\begin{array}{l}\text { chr1:28908266- } \\
\text { 28908287[-] }\end{array}$ & 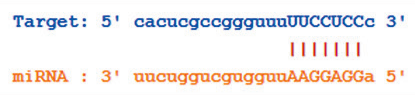 & 7mer-m8 & 4 \\
\hline
\end{tabular}

\section{B}

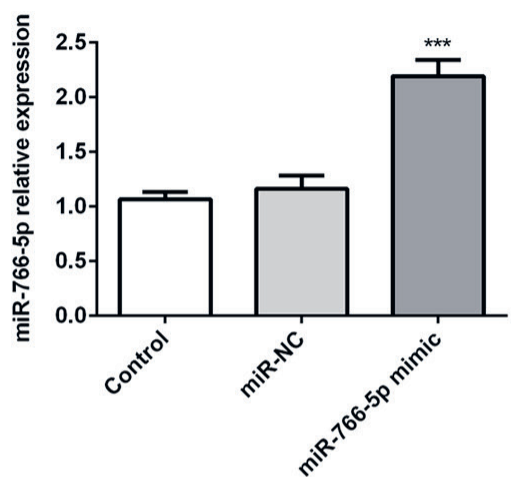

D

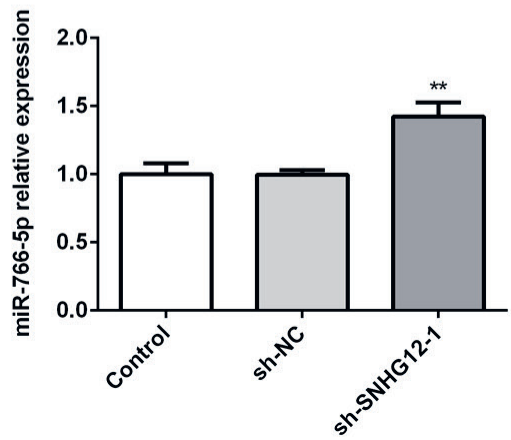

C

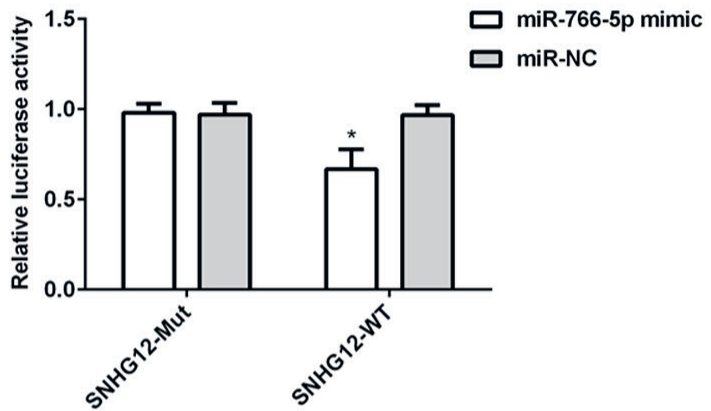

E

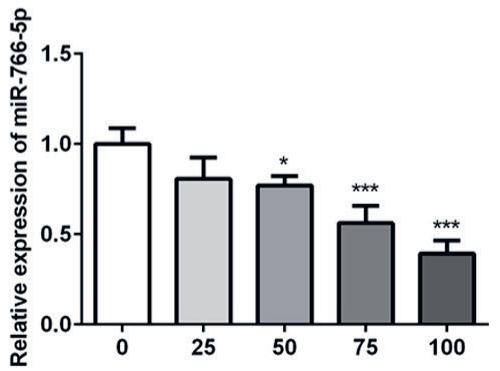

Fig. 2. SNHG12 functions as a sponge of miR-766-5p

A. The potential binding sites were predicted using starBase v. 2.0; B. RT-qPCR was used to determine the expression of miR-766-5p in hVSMCs transfected with miR-766-5p mimic or miR-NC; ${ }^{* * *} p<0.001$ compared to miR-NC group; C. The interaction between SNHG12 and miR-766-5p was validated using luciferase reporter gene assay; ${ }^{*} p<0.05$ compared to miR-NC group; D. The expression of miR-766-5p in hVSMCs transfected with sh-SNHG12-1 or sh-NC; ${ }^{* *} \mathrm{p}<0.01$ compared to the sh-NC group; E. RT-qPCR was used to determine the expression of miR-766-5p in hVSMCs exposed to ox-LDL $(0 \mathrm{mg} / \mathrm{L}, 25 \mathrm{mg} / \mathrm{L}$, $50 \mathrm{mg} / \mathrm{L}, 75 \mathrm{mg} / \mathrm{L}, 100 \mathrm{mg} / \mathrm{L}) ;{ }^{*} \mathrm{p}<0.05,{ }^{* * *} \mathrm{p}<0.001$ compared to $0 \mathrm{mg} / \mathrm{L} \mathrm{ox}-\mathrm{LDL}$.

A
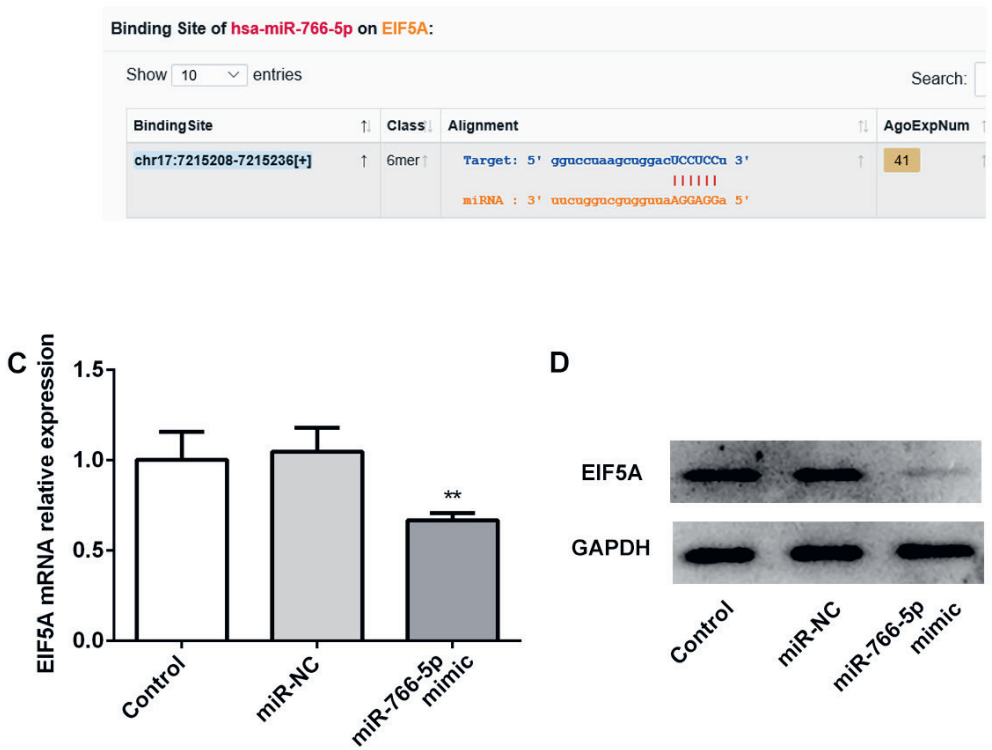

B

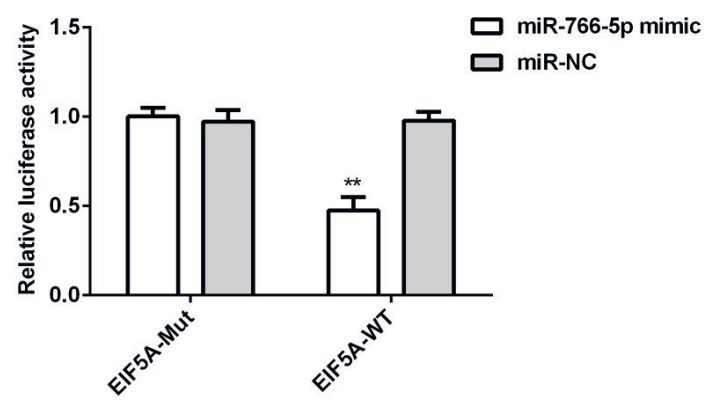

D

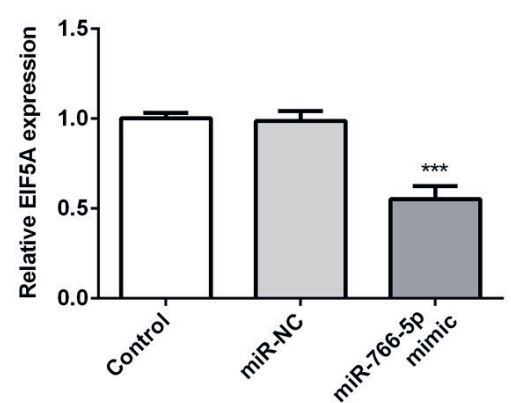

Fig. 3. EIF5A is target gene of miR-766-5p

A. The potential binding sites were predicted using starBase v. 2.0; B. The interaction between miR-766-5p and EIF5A was validated using luciferase reporter gene assay; ${ }^{* *} p<0.01$ compared to miR-NC group; C. RT-qPCR was used to determine the expression of EIF5A in hVSMCs transfected with miR-766-5p mimic or miR-NC; ${ }^{* *} p<0.01$ compared to the miR-NC group; D. western blotting was used to determine the protein levels of EIF5A in hVSMCs; ${ }^{* * *} p<0.001$ compared to the miR-NC group. 

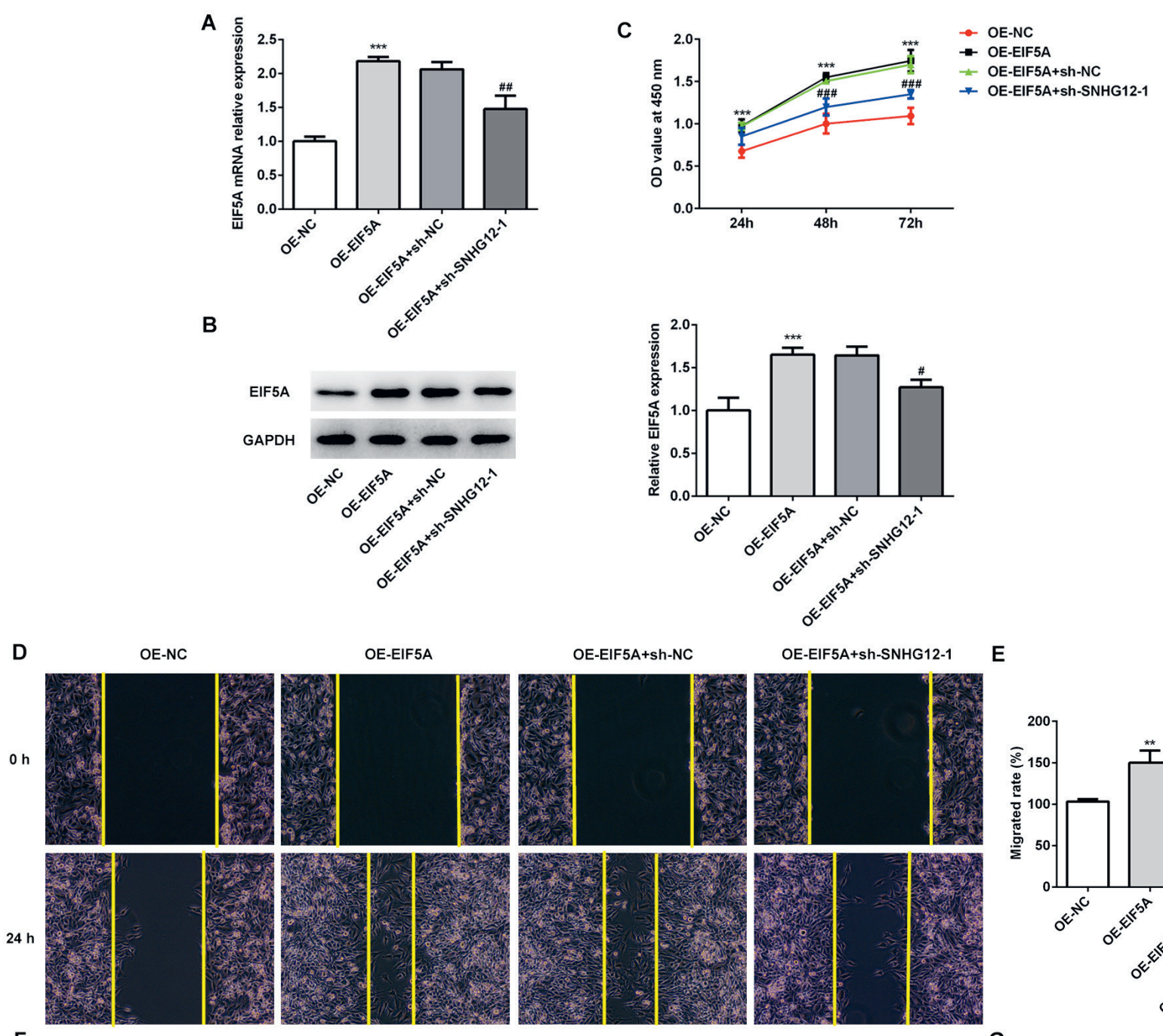

OE-NC

OE-EIF5A

OE-EIF5A+sh-NC

OE-EIF5A+sh-SNHG12-1

$\mathbf{E}$
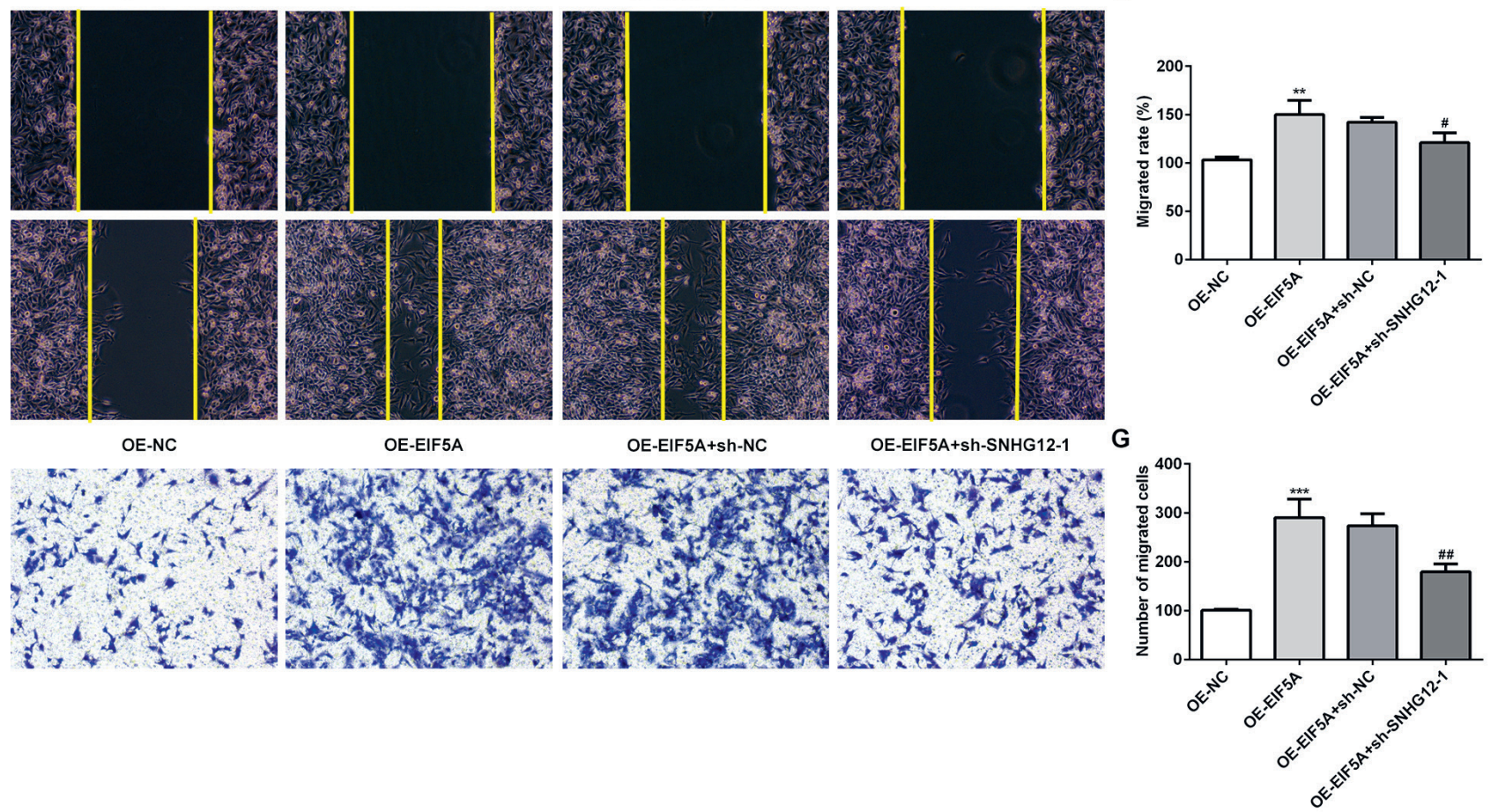

Fig. 4. SNHG12 regulates the proliferation and migration of hVSMCS via mediation of EIF5A

A. The expression of EIF5A was examined using RT-qPCR; ${ }^{* * *} \mathrm{p}<0.001$ compared to the OE-NC group; \#\# $<0.01$ compared to the OE-EIF5A+sh-NC group; B. western blot analysis was used to determine the protein levels of EIF5A in hVSMCs; ${ }^{* * *} \mathrm{p}<0.001$ compared to the OE-NC group; ${ }^{*} \mathrm{p}<0.05$ compared to the OE-EIF5A+sh-NC group; C. The cell viability was estimated using CCK-8 assay; ${ }^{* * *} \mathrm{p}<0.001$ compared to the OE-NC group; \#\#\# $<0.001$ compared to the OE-EIF5A+sh-NC group. The capability of cell migration was assessed using wound healing assay (D and $\mathrm{E})$ and transwell migration assay ( $\mathrm{F}$ and $\mathrm{G}$ ); ${ }^{* *} p<0.01,{ }^{* * *} p<0.01$ compared to the control group; ${ }^{*} p<0.05,{ }^{\# \#} p<0.01$ compared to the ox-LDL+sh-NC group; $\times 100$ magnification.

SNHG12 knockdown exhibited an inhibition of cell migration (Fig. 4D,E). Consistently, transwell migration assays showed a similar result with that of the wound healing assay (Fig. 4F,G). Taken together, these results indicate that SNHG12 mediated the migratory capacities of hVSMCs through regulating EIF5A.

\section{Discussion}

In recent years, numerous studies have demonstrated that lncRNAs regulate various cellular process including cell proliferation, migration, invasion, and apoptosis. ${ }^{24-26}$ It was reported that lncRNA MIAT activates the PI3K/Akt 
signaling pathway, thereby exacerbating atherosclerotic damage in AS mice. ${ }^{3}$ LncRNA activated by transforming growth factor (TGF) expression is significantly higher in AS patients compared with healthy patients, and it could enhance the expression of caspase-3 in human vascular endothelial cells (HUVECs). ${ }^{27}$ Furthermore, the proliferation and migration of VSMCs were promoted by lncRNA $430945 .^{28}$

The hVSMCs are the major cell type observed in blood vessel walls, and play a considerable role in the regulation of multiple physiological and pathological situations. ${ }^{29}$ Aberrant proliferation and migration of VSMCs are key events in the progression of AS and restenosis after percutaneous coronary intervention. ${ }^{30}$ A large amount of studies have suggested that ox-LDL exert a promotion effect in the development of AS by stimulating the proliferation of hVSMCs within the vessel wall; therefore, ox-LDL was widely used to stimulate hVSMCs for investigating the related mechanisms of AS. ${ }^{31,32}$ Studies have implicated SNHG12 in various cancers, and it functions as a potential candidate for cancer-directed interventions. ${ }^{33,34}$ The altered expression of SNHG12 is associated with cell viability, proliferation, metastasis, and invasion, thereby affecting the progression and diagnosis of cancer. ${ }^{13}$ However, the function of SNHG12 in AS has not yet been clearly elucidated. In this study, it was found that ox-LDL facilitated the expression of SNHG12 in hVSMCs. Deletion of SNHG12 impeded cell migration induced by ox-LDL.

Previous reports have described that lncRNAs interact with miRNA as ceRNAs and protect miRNAs from binding to and repressing target RNAs, ${ }^{22,35}$ suggesting a complicated crosstalk among diverse RNA species. Accumulating reports have been made to understand the effect of miRNAs in VSMC biology, especially in cellular proliferation and migration. ${ }^{36-38}$ In our study, luciferase reporter gene assays revealed an interplay between SNHG12 and miR$766-5 p$, and SNHG12 knockdown enhanced the expression of $m i R-766-5 p$. To further examine the target RNA regulated by lncRNA-miRNA, binding sites between $m i R$ 766-5p and EIF5A sequence were predicted using starBase v. 2.0. Subsequently, the interaction between $m i R-766-5 p$ and $E I F 5 A$ was further validated using luciferase reporter gene assay and RT-qPCR. Finally, we found that overexpression of EIF5A expedited the proliferation and migration of hVSMCs, whereas the effect was reversed by SNHG12 silencing.

\section{Conclusions}

The present study illustrates that SNHG12 was highly expressed in ox-LDL-challenged hVSMCs. An intricate interplay among SNHG12, miR-766-5p and EIF5A was discovered, and all of these results indicated that SNHG12knockdown inhibited the proliferation and migration of hVSMCs through targeting the miR-766-5p/EIF5A axis.
Further research is necessary for investigating the impact of SNHG12/miR-766-5p/EIF5A signaling pathway on other pathological alterations in AS progression.

The data supporting our findings are available from the corresponding author upon reasonable request.

\section{ORCID iDs}

Wen Liu (10 https://orcid.org/0000-0003-0629-4305 Jianhuan Che (10) https://orcid.org/0000-0002-9764-8123 Yan Gu (1) https://orcid.org/0000-0003-3952-7611 Ling Song (1) https://orcid.org/0000-0002-3118-2906 Yingying Jiao (1) https://orcid.org/0000-0003-2704-5228 Shui Yu (1) https://orcid.org/0000-0003-1324-8745

\section{References}

1. Geisler S, Coller J. RNA in unexpected places: Long non-coding RNA functions in diverse cellular contexts. Nat Rev Mol Cell Biol. 2013;14(11): 699-712. doi:10.1038/nrm3679

2. Li S, Sun Y, Zhong L, et al. The suppression of ox-LDL-induced inflammatory cytokine release and apoptosis of HCAECs by long non-coding RNA-MALAT1 via regulating microRNA-155/SOCS1 pathway. Nutr Metabol Cardiovasc Dis. 2018;28(11):1175-1187. doi:10.1016/j.numecd. 2018.06.017

3. Sun G, Li Y, Ji Z. Up-regulation of MIAT aggravates the atherosclerotic damage in atherosclerosis mice through the activation of PI3K/ Akt signaling pathway. Drug Deliv. 2019;26(1):641-649. doi:10.1080/ 10717544.2019.1628116

4. Zhou T, Ding JW, Wang XA, Zheng XX. Long noncoding RNAs and atherosclerosis. Atherosclerosis. 2016;248:51-61. doi:10.1016/j.athero sclerosis.2016.02.025

5. Yao X, Yan C, Zhang L, Li Y, Wan Q. LncRNA ENST00113 promotes proliferation, survival, and migration by activating PI3K/Akt/mTOR signaling pathway in atherosclerosis. Medicine (Baltimore). 2018;97(16): e0473. doi:10.1097/MD.0000000000010473

6. Turner AW, Wong D, Khan MD, Dreisbach CN, Palmore M, Miller CL. Multi-omics approaches to study long non-coding RNA function in atherosclerosis. Front Cardiovasc Med. 2019;6:9. doi:10.3389/fcvm. 2019.00009

7. Yang $\mathrm{H}$, Jiang $\mathrm{Z}$, Wang $\mathrm{S}$, et al. Long non-coding small nucleolar RNA host genes in digestive cancers. Cancer Med. 2019;8(18):7693-7704. doi:10.1002/cam4.2622

8. Jin XJ, Chen XJ, Zhang ZF, et al. Long noncoding RNA SNHG12 promotes the progression of cervical cancer via modulating miR125b/STAT3 axis. J Cell Physiol. 2019;234(5):6624-6632. doi:10.1002/ jcp. 27403

9. Cheng G, Song Z, Liu Y, et al. Long noncoding RNA SNHG12 indicates the prognosis of prostate cancer and accelerates tumorigenesis via sponging miR-133b. J Cell Physiol. 2020;235(2):1235-1246. doi:10.1002/ jcp.29039

10. Song J, Wu X, Ma R, Miao L, Xiong L, Zhao W. Long noncoding RNA SNHG12 promotes cell proliferation and activates Wnt/beta-catenin signaling in prostate cancer through sponging microRNA-195. J Cell Biochem. 2019;120(8):13066-13075. doi:10.1002/jcb.28578

11. Zhang R, Liu Y, Liu H, et al. The long non-coding RNA SNHG12 promotes gastric cancer by activating the phosphatidylinositol 3-kinase/ AKT pathway. Aging. 2019;11(23):10902-10922. doi:10.18632/aging. 102493

12. Wu Z, Chen D, Wang K, Cao C, Xu X. Long non-coding RNA SNHG12 functions as a competing endogenous RNA to regulate MDM4 expression by sponging miR-129-5p in clear cell renal cell carcinoma. Front Oncol. 2019;9:1260. doi:10.3389/fonc.2019.01260

13. Tamang S, Acharya V, Roy D, et al. SNHG12: An LncRNA as a potential therapeutic target and biomarker for human cancer. Front Oncol. 2019;9:901. doi:10.3389/fonc.2019.00901

14. Long FQ, Su QJ, Zhou JX, et al. LncRNA SNHG12 ameliorates brain microvascular endothelial cell injury by targeting miR-199a. Neur Regen Res. 2018;13(11):1919-1926. doi:10.4103/1673-5374.238717

15. Yu B, Wang S. Angio-LncRs: LncRNAs that regulate angiogenesis and vascular disease. Theranostics. 2018;8(13):3654-3675. doi:10.7150/ thno. 26024 
16. Wu G, Cai J, Han Y, et al. LincRNA-p21 regulates neointima formation, vascular smooth muscle cell proliferation, apoptosis, and atherosclerosis by enhancing p53 activity. Circulation. 2014;130(17):1452-1465. doi:10.1161/CIRCULATIONAHA.114.011675

17. Wang $S$, Zhang $X$, Yuan $Y$, et al. BRG1 expression is increased in thoracic aortic aneurysms and regulates proliferation and apoptosis of vascular smooth muscle cells through the long non-coding RNA HIF1AAS1 in vitro. Eur J Cardiothorac Surg. 2015;47(3):439-446. doi:10.1093/ ejcts/ezu215

18. Pan JX. LncRNA H19 promotes atherosclerosis by regulating MAPK and NF-kB signaling pathway. Eur Rev Med Pharmacol Sci. 2017;21(2): 322-328. PMID:28165553

19. Livak KJ, Schmittgen TD. Analysis of relative gene expression data using real-time quantitative PCR and the 2(-Delta Delta C(T)) method. Methods. 2001;25(4):402-408. doi:10.1006/meth.2001.1262

20. Cao C, Zhang T, Zhang D, et al. The long non-coding RNA, SNHG6-003, functions as a competing endogenous RNA to promote the progression of hepatocellular carcinoma. Oncogene. 2017;36(8):1112-1122. doi:10.1038/onc.2016.278

21. Jia B, Xia L, Cao F. The role of miR-766-5p in cell migration and invasion in colorectal cancer. Exp Ther Med. 2018;15(3):2569-2574. doi:10. 3892/etm.2018.5716

22. Salmena L, Poliseno L, Tay Y, Kats L, Pandolfi PP. A ceRNA hypothesis: The Rosetta Stone of a hidden RNA language? Cell. 2011;146(3): 353-358. doi:10.1016/j.cell.2011.07.014

23. Mathews MB, Hershey JW. The translation factor elF5A and human cancer. Biochim Biophys Acta. 2015;1849(7):836-844. doi:10.1016/j. bbagrm.2015.05.002

24. Ballantyne MD, Pinel K, Dakin R, et al. Smooth muscle enriched long noncoding RNA (SMILR) regulates cell proliferation. Circulation. 2016; 133(21):2050-2065. doi:10.1161/CIRCULATIONAHA.115.021019

25. Nai Y, Pan C, Hu X, Ma Y. LncRNA LUCAT1 contributes to cell proliferation and migration in human pancreatic ductal adenocarcinoma via sponging miR-539. Cancer Med. 2020;9(2):757-767. doi:10.1002/ cam4.2724

26. Zhang $S$, Chen $P$, Huang Z, et al. Sirt7 promotes gastric cancer growth and inhibits apoptosis by epigenetically inhibiting miR-34a. Sci Rep. 2015;5:9787. doi:10.1038/srep09787

27. Yu H, Ma S, Sun L, Gao J, Zhao C. TGF- $\beta 1$ upregulates the expression of IncRNA-ATB to promote atherosclerosis. Mol Med Rep. 2019;19(5): 4222-4228. doi:10.3892/mmr.2019.10109
28. Cui C, Wang X, Shang XM, et al. IncRNA 430945 promotes the proliferation and migration of vascular smooth muscle cells via the ROR2/ RhoA signaling pathway in atherosclerosis. Mol Med Rep. 2019;19(6): 4663-4672. doi:10.3892/mmr.2019.10137

29. Eun SY, Ko YS, Park SW, Chang KC, Kim HJ. IL-1beta enhances vascular smooth muscle cell proliferation and migration via $\mathrm{P} 2 \mathrm{Y} 2$ receptormediated RAGE expression and HMGB1 release. Vascul Pharmacol. 2015;72:108-117. doi:10.1016/j.vph.2015.04.013

30. Fan TF, He JH, Yin YQ, et al. Dioscin inhibits intimal hyperplasia in rat carotid artery balloon injury model through inhibition of the MAPKFoxM1 pathway. Eur J Pharmacol. 2019;854:213-223. doi:10.1016/j. ejphar.2019.03.050

31. Pirillo A, Norata GD, Catapano AL. LOX-1, OxLDL, and atherosclerosis. Mediators Inflamm. 2013;2013:152786. doi:10.1155/2013/152786

32. Yang N, Dong B, Song YQ, et al. Downregulation of miR-637 promotes vascular smooth muscle cell proliferation and migration via regulation of insulin-like growth factor-2. Cell Mol Biol Lett. 2020;25:30. doi:10.1186/s11658-020-00222-z

33. Wang O, Yang F, Liu Y, et al. C-MYC-induced upregulation of IncRNA SNHG12 regulates cell proliferation, apoptosis and migration in triple-negative breast cancer. Am J Trans/ Res. 2017;9(2):533-545. PMID: 28337281

34. Zhang $\mathrm{H}$, Lu W. LncRNA SNHG12 regulates gastric cancer progression by acting as a molecular sponge of miR320. Mol Med Rep. 2018;17(2): 2743-2749. doi:10.3892/mmr.2017.8143

35. Tay Y, Rinn J, Pandolfi PP. The multilayered complexity of ceRNA crosstalk and competition. Nature. 2014;505(7483):344-352. doi:10. 1038/nature12986

36. Choe N, Kwon JS, Kim JR, et al. The microRNA miR-132 targets Lrrfip1 to block vascular smooth muscle cell proliferation and neointimal hyperplasia. Atherosclerosis. 2013;229(2):348-355. doi:10.1016/j. atherosclerosis.2013.05.009

37. Li FP, Lin DQ, Gao LY. LncRNA TUG1 promotes proliferation of vascular smooth muscle cell and atherosclerosis through regulating miRNA21/PTEN axis. Eur Rev Med Pharmacol Sci. 2018;22(21):7439-7447. doi:10.26355/eurrev_201811_16284

38. Huang SC, Wang M, Wu WB, et al. Mir-22-3p inhibits arterial smooth muscle cell proliferation and migration and neointimal hyperplasia by targeting HMGB1 in arteriosclerosis obliterans. Cell Physiol Biochem. 2017;42(6):2492-2506. doi:10.1159/000480212 